\title{
80/10Gb/s Downstream/Upstream Capacity Multi-wavelength TDM-PON
}

\author{
Lilin Yi, Zhengxuan Li,Yi Dong, Shilin Xiao and Weisheng Hu \\ State Key Lab of Advanced Optical Communication Systems and Networks, Shanghai Jiao Tong University, \\ Department of Electronic Engineering, Shanghai 200240, China \\ *Corresponding author: lilinyi@sjtu.edu.cn
}

\begin{abstract}
This paper proposes a novel multi-wavelength time division multiplexing passive optical network (TDMPON) configuration using a single tunable optical filter (TOF) for both downstream wavelength selection and upstream wavelength generation. The network architecture is completely compatible with the existing optical distribution network and can also mitigate Rayleigh backscattering and back reflection induced crosstalk effectively. A total capacity of $8 \times 10 \mathrm{~Gb} / \mathrm{s}$ for downstream and $8 \times 1.25 \mathrm{~Gb} / \mathrm{s}$ for upstream has been demonstrated with a $25 \mathrm{~km}$ feeder fiber and 1:32 splitting ratio.
\end{abstract}

Keywords- Multi-wavelength TDM-PON; Tunable optical filter (TOF);RSOA;Rayleigh backscattering.

\section{INTRODUCTION}

Passive optical network (PON) has been believed as a good solution to provide an economic implementation of broadband access, in which time division multiplexing (TDM) based GPON and EPON have been standardized and mass-deployed. In the TDM-PON, a single wavelength channel is shared by all users with the help of a splitter in the remote node (RN). However, the capacity of a single wavelength has difficulty in accommodating the upcoming subscribers' demand due to the blossom of various Internet services. Meanwhile, as the access network is cost-sensitive, it's of great concern for network operators to provide a cost-effective, smooth upgrade which could realize a bandwidth expansion without making major changes to the already deployed optical distribution network (ODN). Stacked TDM-PON or multi-wavelength TDM-PON has captured a lot of research interest during the past years [1, 2], which provides a promising solution for smooth capacity upgrade by overlaying multiple wavelengths on the existing TDM-PON.

Downstream wavelength selection and upstream laser generation are two key problems to be solved in the stacked TDM-PON proposal. As no arrayed waveguide grating (AWG) is deployed in the RN, the downstream wavelength selection should be realized in the optical network unit (ONU). Besides, the ONU requires a lowcost tunable laser source for multiple upstream wavelengths generation. In previous researches, the downstream wavelength selection was always realized by using an optical filter in the ONUwhile the options for upstream wavelength generation are various. FP-LD and Reflective semiconductor optical amplifiers (RSOA) are the most common candidates for upstream laser source due to their low cost and the ability to be directly modulated. Limited by the narrow spectral bandwidth and the fixed wavelength spacing between two neighboring modes in the FP-LD, the laser source has a wavelength tuning range of less than $20 \mathrm{~nm}$ and a tuning step of $\sim 1 \mathrm{~nm}$. Besides, the power ripple among different channels is higher than $5 \mathrm{~dB}$, which limits the power budget of the upstream link. RSOA based laser source has a wider tuning range and a more flexible tuning character than FP-LD, on which a lot of researches have been reported [3]. But as the RSOA is seeded by the arrayed lights in the optical line terminal (OLT), the wavelength number and tuning flexibility are determined by the centralized laser bank, which increases the cost of OLT. Besides, Rayleigh backscattering and back reflection induced crosstalk degrades the upstream signal quality severely in the centralized laser source case [4].

We have previously proposed a compatible TDM/ wavelength division multiplexing (WDM) PON architecture using a RSOA and tunable optical filter (TOF) based directly modulated tunable fiber ring laser as upstream laser source [5]. A TOF in the ONU selects downstream wavelengths and also plays a secondary role of seeding the RSOA in a fiber ring cavity for upstream laser generation, realizing a smooth upgrade from TDMPON to a hybrid TDM/WDM-PON without making major change to the existing ODN. The self-phase modulation (SPM) induced spectral red shift in the saturated RSOA results in a wavelength offset between downstream and upstream wavelengths, which could effectively eliminate the back reflection and Rayleigh backscattering induced power penalty. In this paper, we further investigated the multi-wavelength transmission capability of the proposed network architecture and evaluated the performance of the system. 8 wavelengths bidirectional transmission with $10 \mathrm{~Gb} / \mathrm{s}$ for downstream and $1.25 \mathrm{~Gb} / \mathrm{s}$ for upstream per wavelength over a single fiber is successfully demonstrated with almost no power penalty at both ends and tiny differences in sensitivities. It is also verified that the shape and bandwidth of the TOF do not affect the output power and the transmission performance of upstream laser source; therefore the laser source can be easily implemented with low cost solution. The power budget has been valued to be around $16 \mathrm{~dB}$, which could support 1:32 splitting with a $25 \mathrm{~km}$ feeder. 


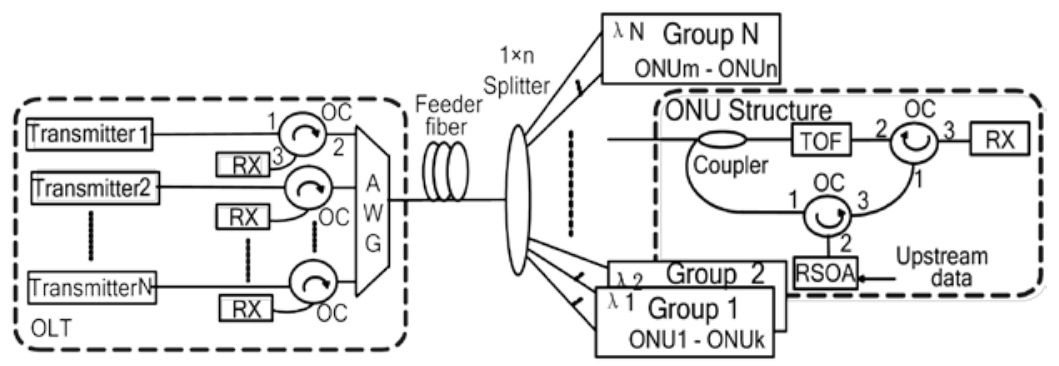

Fig.1 Stacked TDM-PON configuration

\section{STACKED TDM-PON ARCHITECTURE}

The configuration of the proposed stacked TDMPON is shown in Fig.1. In the downstream direction, an array of lasers multiplexed by an AWG is assigned in the OLT as downstream sources. After fiber transmission, the downstream signal is broadcasted to all ONUsbya $1 \times$ nsplitter. Then it passes through an optical filter whose central wavelength is aligned with one of the downstream wavelengths for selection and finally detected by the receiver. In the upstream link, the TOF used for downstream wavelength selection is connected to an RSOA, thereby forming a fiber ring laser whose output wavelength is controlled by tuning the central wavelength of the TOF. By driving the RSOA into its saturated region, data can be directly applied to the RSOA for modulation [6]. The coupler in the ONU launches partial of the laser output into the splitter for multiplexing and after transmission the upstream signal is demultiplexed by the AWG in the OLT and finally detected by the receiver. In the network configuration, all ONUs are divided into $\mathrm{N}$ groups, each group owns a single wavelength and ONUs in the same group share the same wavelength in TDM way by tuning their TOFs on the same wavelength.

\section{EXPERIMENTAL SETUP AND RESULTS}

\section{A. Experimental setup}

We made an experiment to investigate the performance of the proposed scheme as shown in Fig.2.At the OLT side, a Mach-Zehnder modulator (MZM) driven by a $10-\mathrm{Gb} / \mathrm{s}$ Pseudo-random bit sequence (PRBS)data is usedto modulate the downstream laser generated by a tunable laser source (TLS). After $25-\mathrm{km}$ single mode fiber (SMF) transmission, the signal passes through a coupler, aTOF, OC2 and finally detected by an optical receiver. The TOFis a super-Gaussian filter with a bandwidth of $0.8 \mathrm{~nm}$. The central wavelength of the TOF is aligned to the downstream wavelength for a minimal loss. In the upstream link, the laser source consists of a

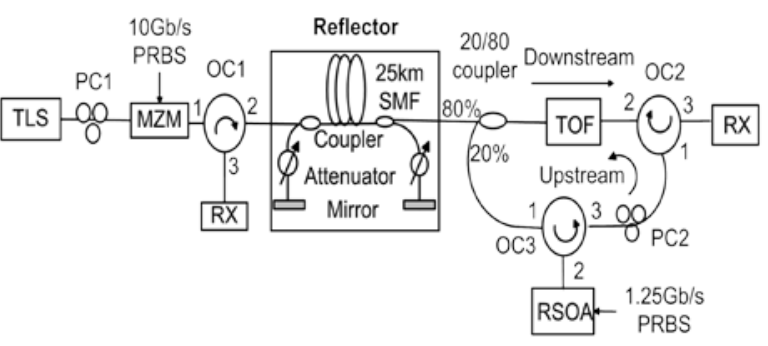

Fig.2 Experimental setup

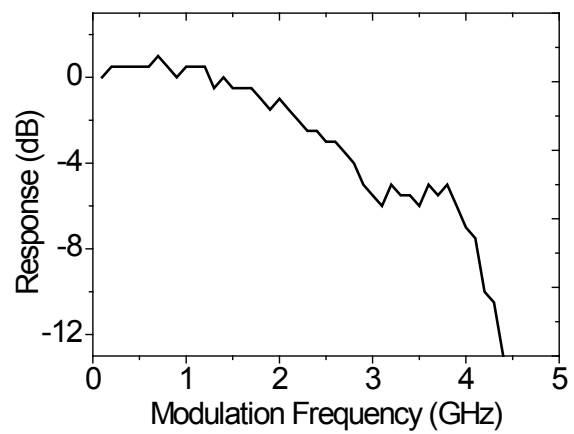

Fig.3 The frequency response of the RSOA

20/80 coupler, a TOF, two optical circulators, a polarization controller and a RSOA. PRBS data is directly applied to the RSOA as upstream signal.The RSOA has a modulation speedlimited by photon density. We measured the frequency response of the RSOA as shown in Fig. 3. The 3-dB bandwidth of the RSOA is about 2.5GHz.Noted that the measured frequency response is the combination between the RSOA itself and the data driver.

\section{B. Performance of upstream laser source}

The performance of the upstream laser source is firstly investigated. As we tune the central wavelength of the TOF, the output wavelength changes accordingly. Fig.4 (a) shows the measured optical spectra measured within the tuning range of the laser source from $1530 \mathrm{~nm}$ to $1595 \mathrm{~nm}$ with a $5 \mathrm{~nm}$ tuning step. The output powers have a fluctuation less than $2 \mathrm{~dB}$ within the whole tuning range. It's interesting we observed that the laser generated in the cavity always locates at the long wavelength side within the passband of the filter due to the SPM induced spectral red-shift effect in the saturated RSOA [7], as shown in the inset of Fig.4 (b). It will be specified in the following contents that with this wavelength offset, the back reflection and Rayleigh backscattering induced crosstalk can be effectively mitigated. We modulate the RSOA with a $1.25 \mathrm{~Gb} / \mathrm{s}$ PRBS and measured the BERs of the upstream signal both in back-to-back (BtB) case and after 25-km SMF transmission. The results are shown in Fig.4 (b). The sensitivity of the upstream signal is about $-30 \mathrm{dBm}$ and almost no power penalty wasobservedafter $25 \mathrm{~km}$ SMF unidirectional transmission. 

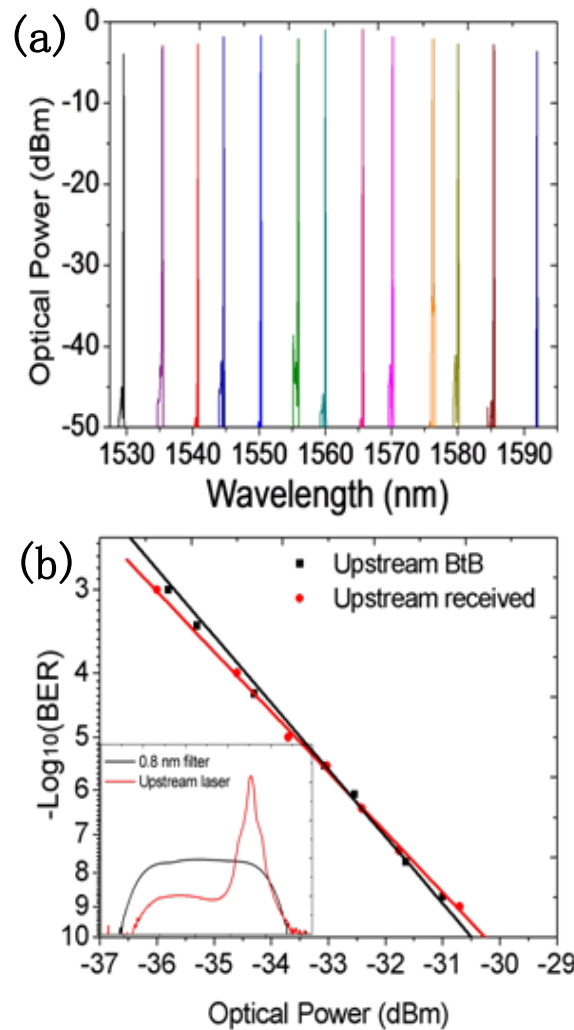

Fig.4 Performance of the proposed upstream laser source

All the results above show that laser source features atuning range from $1530 \mathrm{~nm}$ to $1595 \mathrm{~nm}$, an output power higher than $-3 \mathrm{dBm}$, a power ripple lessthan $2 \mathrm{~dB}$, SMSRhigh than $60 \mathrm{~dB}$, which perfectly meets the requirement of the laser source in the upgraded ONU.

\section{Rayleigh backscattering elimination}

We tested the transmission performance of the proposed configuration. As mentioned above, the offset between upstream laser and central wavelength ofthe TOF provides a good chance to eliminate the back reflection and Rayleigh backscattering induced crosstalk in single fiber bidirectional transmission. To clarify thispoint, we added reflectors at both ends of the feeder fiber and demonstrated a single fiber bidirectional transmission.The eye diagrams and BERs of both downstream and upstream signals under various reflected noise to signalratio (RSNR) and wavelength difference cases are measured, the results are shown in Fig.5. The eye diagrams of both directions are distorted severely and cannot be detected when the downstream and upstream signals have the exact same wavelength. But when the upstream wavelength is tuned away from the downstream one, the eyes become clear again. A $0.2-\mathrm{nm}$ difference between downstream and upstream wavelengths is able to reduce the crosstalk induced power penalty to less than 1 $\mathrm{dB}$ compared with the BtB case when the RNSR is set up to $12 \mathrm{~dB}$. As we tune the central wavelength of the TOF from $1530 \mathrm{~nm}$ to $1595 \mathrm{~nm}$, similar results are observed, proving the back-reflection induced crosstalk suppression method is feasible for practical application.

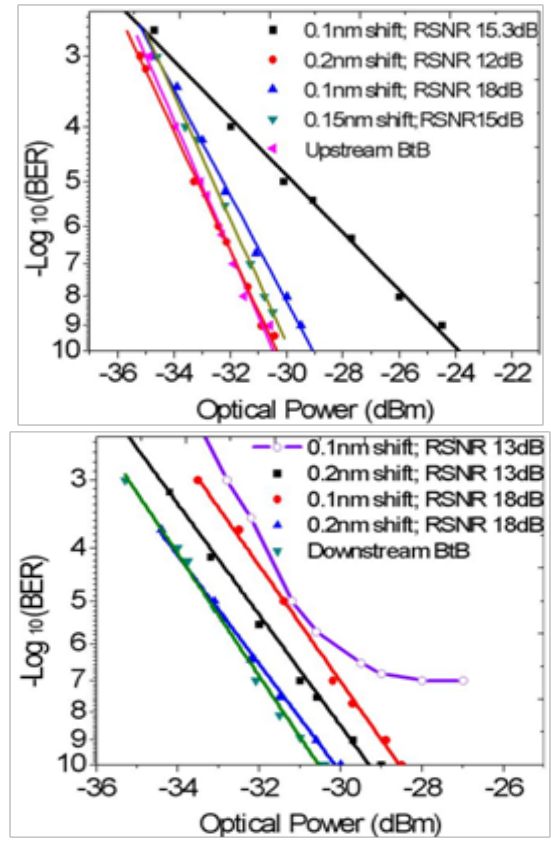

Fig.5 Measured BER of downstream and upstream signals in $\mathrm{BtB}$ and bidirectional transmission

\section{D. $80 / 10 \mathrm{~Gb} / \mathrm{s}$ capacity demonstration}

To evaluate the performance of the system in multiwavelength application, an 8 wavelength stacked experiment demonstration is performed in detail. The experimental setup for 8 wavelengths stacked TDM-PON is given in Fig.6. The downstream is implemented by using 8 distributed-feedback (DFB) lasers with each one aligned to one channel of the AWG. At the ONU side, 8 sets of ONUs are multiplexed by a $1 \times 8$ splitter. Similar with the downstream link, the passband of TOF in each $\mathrm{ONU}$ is tuned to match the AWG channels. Downstream lasers are all modulated by $10 \mathrm{~Gb} / \mathrm{s}$ PRBS while RSOAs in each ONU is modulated by $1.25 \mathrm{~Gb} / \mathrm{s}$ PRBS data. We measured the BERs of signals on all the channels and Fig.7 gives the sensitivities in BtB and after transmission cases at both ends. After transmission, almost no power penalty is observed in both directions. These curves also indicate that the upstream signal has an even lower sensitivity fluctuation of $\sim 1 \mathrm{~dB}$ than the downstream signal of $\sim 2 \mathrm{~dB}$, verifying the stability of the upstream laser source.

To verify the maximal user's number this system could support, a power budget calculation was carried out, which is determined by the upstream laser bank. As shown in Fig.3, the lowest output power of upstream laser is $-3 \mathrm{dBm}$ and the worst sensitivity of received upstream signal is $-29 \mathrm{dBm}$ as Fig.7 shows. If we consider the AWG insertion loss of $4 \mathrm{~dB}$ and the measured attenuation of $25 \mathrm{~km}$ fiber by $5 \mathrm{~dB}$, with the addition of other connector losses of $1 \mathrm{~dB}$, the remaining budget is $16 \mathrm{~dB}$. This can be used to insert a 1:32 splitter towards ONUs at the RN. For further enhancement, the output power can be higher if a higher gain RSOA is used. And the receiver sensitivity can be optimized if a $1.25 \mathrm{~Gb} / \mathrm{s}$ rather than a $10 \mathrm{~Gb} / \mathrm{s}$ photo-detector is used for upstream detection. 


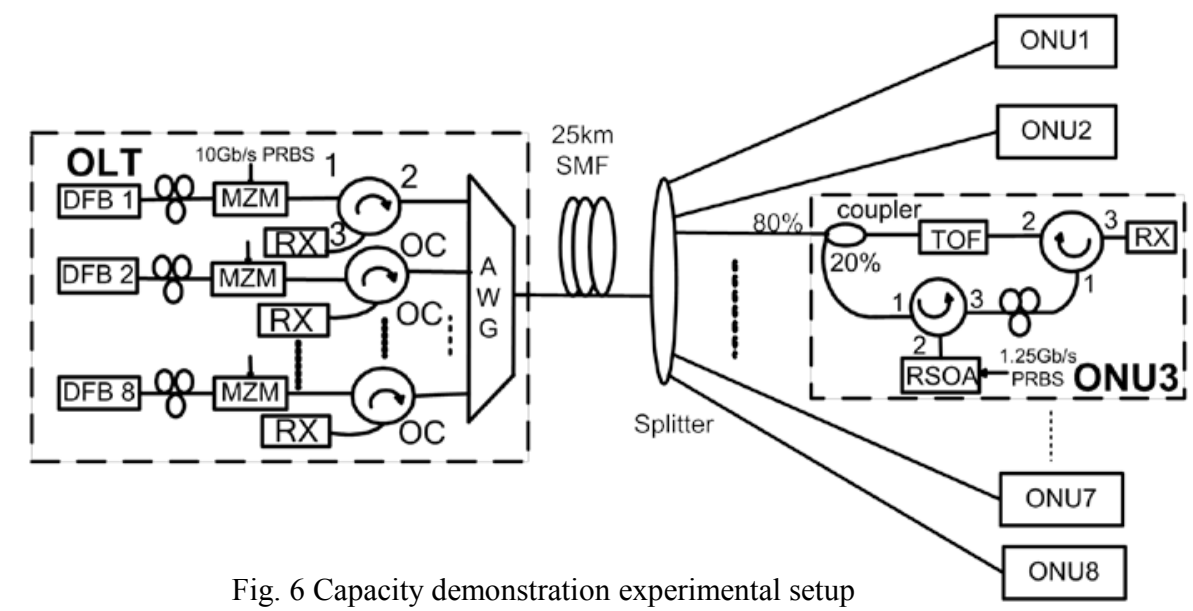

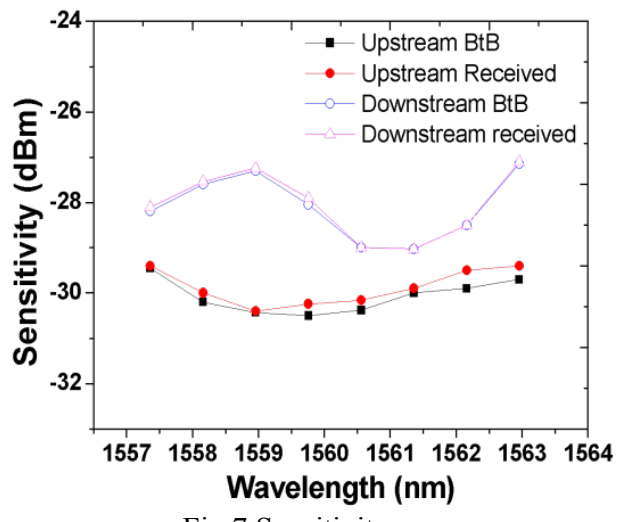

Fig.7 Sensitivity curves

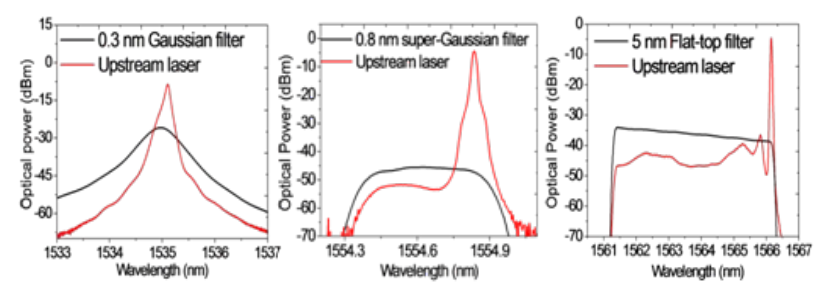

Fig.8 Spectra of upstream laser and passband of TOF

Finally, the cost of the proposal is evaluated, as other devices are of certain characters, optical filter is the only controllable element that has the potential to simplify the ONU structure and lower down the cost of each user. In order to define the characters of the TOF requested for laser source construction, we varied the width and shape of the TOF's passband and it turns out that no matter the filter is of Gaussian, super-Gaussian or flat-top shape, or the bandwidth ranges from $0.3 \mathrm{~nm}$ to 5 $\mathrm{nm}$, the laser can always be generated with almost no power difference as shown in Fig.8. The sensitivities of signal modulated on the lasers are also proved to be similar. In this way, as requirement on the TOF is not rigid, the TOF can be manufactured using simple technologies therefore reducing the cost.

\section{CONCLUSIONS}

We proposed a stacked TDM-PONconfigurationwhich could upgrade the capacity of the traditional TDM-PON greatly without making major changes to the existing ODN. A RSOA and TOF based directly modulated tunable fiber ring laser performs as upstream laser source while the TOF bears a second role of selecting wavelength for each ONU.Request on the TOF was proved to be relaxed so that the laser source can be easily implemented. The red shift feature of the upstream laser source enables a difference between upstream and downstream wavelengths, which can effectively mitigate the back reflection and Rayleigh backscattering induced crosstalk. A total capacity of $8 \times 10 \mathrm{~Gb} / \mathrm{s}$ for downstream and $8 \times 1.25 \mathrm{~Gb} / \mathrm{s}$ for upstream has been demonstrated with a $25 \mathrm{~km}$ feeder fiber and 1:32 splitting ratio.

\section{ACKNOWLEDGMENT}

This work was supported by 973 Program (2012CB315602, 2010CB328204-5), Nature Science Foundation China (61007041, 61090393, 61132004 and 60825103), 863Program, Program of Shanghai Subject Chief Scientist (09XD1402200), Program of Shanghai Chen Guang Scholar (11CG11) and Program of Excellent $\mathrm{PhD}$ in China (201155).

\section{REFERENCES}

[1] R. Heron, "Next Generation Optical Access Networks," in Access Networks and In-house Communications, OSA Technical Digest (CD) (Optical Society of America, 2011), paper AMA2.

[2] F. J. Effenberger, "10G PON - Explained," in Optical Fiber Communication Conference, OSA Technical Digest (CD) (Optical Society of America, 2010), paper OWX4

[3] T. Jayasinghe, C. J. Chae, and R. S. Tucker, "Scalability of RSOA-based multi-wavelength Ethernet PON architecture with dual feeder fiber," J. Opt. Netw. 6, 1025-1040 (2007).

[4] Patryk J. Urban, A. M. J. Koonen, G. DjanKhoe, and Huug de Waardt "Interferometric Crosstalk Reductionin an RSOA-Based WDM Passive Optical Network", J. Lightw. Technol, 27, 49434953, (2009).

[5] Zhengxuan Li, Lilin Yi, Yan Zhang, Yi Dong, Shilin Xiao and Weisheng $\mathrm{Hu}$, "Compatible TDM/WDM PON using a Single Tunable Optical Filter for both Downstream Wavelength Selection and Upstream Wavelength Generation," to be published on Photon. Technol.Lett.

[6] Zih-Rong Lin, Cheng-Kuang Liu, Yu-JhuJhang, and Gerd Keiser, "Tunable directly modulated fiber ring laserusing a reflective semiconductor optical amplifier for WDM access networks," Opt Express,18, 17610-17619, (2010).

[7] G. P. Agrawal and N. A. Olsson, "Self-phase modulation and spectral broadening of optical pulses in semiconductor laser amplifiers," IEEE J. Quantum Electron, 25, 2297-2306, (1989). 